\title{
Minireview
}

\section{Differential proteomic alterations between localised and metastatic prostate cancer}

\author{
BS Taylor ${ }^{1,2}$, S Varambally ${ }^{1,3}$ and AM Chinnaiyan ${ }^{*, 1,2,3,4}$ \\ 'Department of Pathology, University of Michigan Medical School, Ann Arbor, Ml 48109, USA; ${ }^{B}$ Bioinformatics Program, University of Michigan Medical \\ School, Ann Arbor, MI 48109, USA; ${ }^{3}$ Comprehensive Cancer Center, University of Michigan Medical School, Ann Arbor, MI 48 I 09, USA; ${ }^{4}$ Department of \\ Urology, University of Michigan Medical School, Ann Arbor, MI 48109, USA
}

\begin{abstract}
Molecular alterations in the prostate cancer proteome mediate the functional and phenotypic transformation from clinically localised to metastatic cancer, a transition that drives patient's mortality and challenges therapeutic intervention. A first approximation of differential proteomic alterations stratified by disease stage has yielded repertoires of potential diagnostic and prognostic markers, multiplex signatures of predictive value, and yield fundamental insight into molecular commonalities in cancer progression. Deciphering these causative proteomic alterations from the molecular noise will continue to mature our understanding of tumour biology and drive new computational and integrative approaches to model a system's view that accommodates the heterogeneity of prostate cancer progression.
\end{abstract}

British Journal of Cancer (2006) 95, 425-430. doi: I0.1038/sj.bjc.6603274 www.bjcancer.com

Published online I August 2006

(c) 2006 Cancer Research UK

Keywords: metastasis; cancer proteomics; bioinformatics; systems biology; prostate cancer; tumour marker

Although clinically localised prostate cancer can be treated with androgen ablation, surgical resection or radiation, its transition to metastatic disease is almost uniformly fatal. Understanding the molecular hallmarks of this transition has been the target of extensive study by the wider research community. In this context, a multitude of both diagnostic and prognostic tissue and serum biomarkers have been proffered as viable supplements to the standard clinical parameters in use now. These include prostatespecific antigen, Gleason score and clinical stage; however, these are individually opaque to sensitive disease fate decisions. In parallel, there are emerging efforts in integrative and systems approaches to prostate cancer progression. These promise not only molecular profiles and signatures, but also models of progression that assimilate the heterogeneity and complexity of a decidedly continuous and nonlinear biological process. We focus here on recent approaches and discoveries, and specifically in the context of those proteomic alterations between the later, progressive stages of prostate cancer.

\section{DISCOVERY AND DIVERSITY OF ALTERATION TYPES AND A VECTOR OF PROTEOMIC CHANGE}

The explosion of high-throughput platforms for protein-level analysis, primarily driven by mass spectrometry and array-based methodologies, represents a transition to an exponential phase in oncoproteomics. The biomedical literature is replete with prostate

\footnotetext{
*Correspondence: Dr AM Chinnaiyan, Department of Pathology, University of Michigan Medical School, 130 I Catherine Rd, MSI Rm 4337, Ann Arbor, MI 48109-0602, USA; E-mail: arul@umich.edu Received 24 April 2006; revised 9 June 2006; accepted 23 June 2006; published online I August 2006
}

cancer profiling efforts and biomarker discovery in serum, urine, and a growing number in tissue along with variations on early detection platforms and technologies (Wulfkuhle et al, 2003). Many of these are exploiting the more immediate translational opportunities in clinical proteomics. These include biofluid profiling, despite the challenges of reproducibility from physiological variability. As our focus here is on proteomic alterations in the prostate cancer tissue proteome, we benefit from circumventing the many problems of secondary and tertiary clinical conditions that drive this physiological variation between and among patient cohorts. Alternatives exist to these explicit and discrete protein biomarker identification techniques. One such method is SELDI-TOF-derived proteomic pattern diagnostics coupled to both significant bioinformatics and up- and downstream sample processing. Nevertheless, much of the existing work across all sample classes confirms a confounding paradox in the cancer proteomics community. Although the vast majority of FDAapproved biomarkers are indeed proteins, none were discovered via high-throughput experimentation and few are used in routine clinical practice (Ludwig and Weinstein, 2005). Consequently, work of our group and others focuses on satisfying the twin and overlapping goals of viable biomarker discovery and deconvoluting the proteomic mechanisms in prostate cancer progression.

During the stages of cancer progression, the tissue proteome fluctuates exquisitely reflecting a dynamism fundamental to the complexity of the process. It is a constantly moving target, the combination of changes that span a vast array of both physical and functional modifications, as well as proteins being secreted and culled from the circulating proteome or degraded by deregulated proteolytic processes. Modifications include differential expression and protein coexpression, most frequently quantified in clinical samples with chemically incorporated stable isotope labelling coupled to high mass accuracy mass spectrometry. Another is altered localisation characterised by the direct analysis of tissue 
sections with methods such as MALDI imaging mass spectrometry (IMS) (Caprioli, 2005). Other changes include modifications to networks of protein-protein interactions mediating nearly all of the molecular events in disease progression, which are amenable to protein microarray analysis. Additionally, and playing a pivotal functional role in oncogenesis and progression, are post-translational modifications, which require platforms of high mass accuracy and increasing levels of sensitivity for high-throughput discovery. Finally, it is worth mentioning the effect that isolation and extraction techniques, such as laser-capture microdissection (LCM), are having on the known altered proteome in prostate tissue, whether clinically localised or metastatic disease. Althoughly this method aids immeasurably in generating homogenous tumour cell populations for study and certainly improves the specificity of electrophoretic methods like 2D-PAGE in biomarker discovery, it may also help unmask critical alterations otherwise unavailable at the whole-tissue level. The tumour microenvironment is a heterogeneous mix of cell types that includes stromal constituents. In lieu of pure epithelial cell models, LCM in combination with better computational tools is helping to sift out functionally contributing stromal proteins from those that are truly contamination. This will capture a more complete picture of the proteomic interplay in the tumour microenvironment that includes potentially important stromal components. Together, these technologies are generating individual and combinations of proteomic targets.

Although rigid protein repertoires generated from these platforms are informative, they are single dimensional. As a result, their sufficiency is subordinate to otherwise factorial molecular behaviour. Much of the newest proteomic profiling work is generating steady-state first approximations of the differential proteome between discrete stages of prostate cancer (Varambally et al, 2005). Furthermore, this work represents an analytical shift towards integrative molecular study, a systems approach to dissecting the molecular events that dictate prostate cancer progression. In this context, there are significant qualitative changes happening both locally and globally at the proteomic level, independent of any single protein, which might be thought of as gross proteomic remodelling between disease stages.

Our group and others have observed, via high-throughput tissue microarray analysis, a general vector of qualitative change at the protein level between clinically localised and metastatic tissue extracts. Leveraging immunohistochemically compatible antibo- dies, and aside from differences in individual alterations, we are witnessing broader trends in epithelial cell staining paralleling discrete transitions in progressive disease (Varambally et al, 2005). This includes metastatic tumours showing reduced membranous staining as compared to localised tumours, which might be predicted by canonical models of adhesion, invasion and proteolytic degradation. Further, these may become increasingly accessible to characterisation as methods for mass spectrometrybased membrane proteomics improve (Wu and Yates, 2003). Additionally, cellular conflation and tumour composition also affect proteomic behaviour, which is uncovered in subsequent staining. This is attributable to factors including the increase in density of tumour cells potentiating higher protein expression levels in localised and metastatic prostate cancer, as well as shifts in stromal to epithelial components between disease stages. Tumours also demonstrate variable levels of subcellular and, especially, nuclear protein expression across a range of markers, which may again be a product of cellular density and not necessarily a direct product of tumour progression. These higher-level observations purport a holistic approach to tissue proteome analysis. Vertically integrating between the depths of analysis in individual protein alterations to the higher-level breadth of gross behavioural observations allows for larger and more comprehensive models of the prostate cancer tissue proteome.

\section{ALTERATIONS IN A GLOBAL PROGRESSION SIGNATURE}

There is currently a relative paucity of work on profiling proteomic alterations in tissues between the various disease stages in prostate cancer. This is more often dealt with at the transcriptomic level (Stanbrough et al, 2006), from which the vast majority of existing prostate cancer markers, prognostic or otherwise, have been characterised. However, the newest parallel efforts in systematic protein analysis are further elucidating the behaviour of these and many more, of which we have sampled a small fraction (Table 1). A portion of these is extensively well-studied and fundamentally archetypal of high-throughput profiling for disease markers and are well-reviewed elsewhere (Kumar-Sinha and Chinnaiyan, 2003). Nevertheless, we spend some time here discussing a subset of these; a transcriptional repressor, enzyme, kinase and two nuclear

Table I Limited sampling of protein alterations in clinically localised prostate cancer relative to benign prostate and metastatic prostate cancer relative to clinically localised

\begin{tabular}{|c|c|c|c|c|}
\hline Locus & \multicolumn{2}{|c|}{ Alteration } & Prognostic & Reference \\
\hline AMACR & $\uparrow\left(\uparrow^{\mathrm{a}}\right)$ & $\downarrow\left(\downarrow^{\mathrm{a}}\right)$ & $Y \mid N$ & Rubin et al (2002) \\
\hline HEPSIN & 个 $\left(\boldsymbol{\uparrow}^{\mathrm{a}}\right)$ & $\downarrow\left(\downarrow^{a}\right)$ & - & Dhanasekaran et al (200I) \\
\hline $\mathrm{EZH} 2$ & $\Leftrightarrow(\uparrow)$ & $\uparrow(\uparrow)$ & Y & Varambally et al (2002) \\
\hline STKI5 & $\Leftrightarrow(\uparrow)$ & $\uparrow(\uparrow)$ & Y & Jeng et al (2004) \\
\hline MET & $\uparrow\left(\downarrow^{a}\right)$ & $\uparrow\left(\downarrow^{\mathrm{a}}\right)$ & $N$ & Watanabe et al (1999) \\
\hline pPKR & $-^{b}(-)$ & $\downarrow^{c}(-)$ & - & Jammi and Beal (200I) \\
\hline ICBP90 & $\Leftrightarrow(\Leftrightarrow)$ & $\uparrow(\uparrow)^{\prime}$ & - & Hopfner et al (2000) \\
\hline BM28 & $\uparrow(\Leftrightarrow)$ & $\uparrow(\uparrow)$ & $Y$ & Meng et al (200I) \\
\hline $\mathrm{MSH} 2$ & $\uparrow(\uparrow)$ & $\uparrow(\uparrow)$ & - & Velasco et al (2002) \\
\hline UBC9 & $\uparrow(\Leftrightarrow)$ & $\uparrow\left(\uparrow^{\mathrm{a}}\right)$ & - & Poukka et al (1999) \\
\hline
\end{tabular}

Arrows and weights reflect directionality and degree of change in protein expression. mRNA transcript expression is included as a reference and to emphasise the multidimensionality of integrative analysis of protein alterations. Additional annotations include the superscript. ${ }^{a}$ For oncomine-derived expression across multiple prostate cancer profiling studies (Rhodes et al, 2004). ' Represents phosphorylation. ' Represents post-translational modifications of any kind. 
proteins, which serve as an example of the multi-dimensionality of proteomic alterations between stages of prostate cancer. Additionally, that all of these were discovered or recapitulated by our laboratory's recent work with a single high-throughput immunoblot approach emphasises the capacity of new experimental and integrative approaches to model large swaths of the differential prostate cancer proteome (Varambally et al, 2005).

The first of these proteins is EZH2, the human homologue of the Drosophila protein Enhancer of Zeste $(\mathrm{E}(\mathrm{Z}))$, a member of the Polycomb group of proteins. It is involved in epigenetic gene silencing. Recently, it has been linked via DNA methyltransferases, suggesting a direct connection between two key epigenetic repression systems (Vire et al, 2006). Our group evaluated the expression of EZH2 by immunohistochemistry using tissue microarrays from patients with either clinically localised or hormone-refractory prostate cancer. The result indicated poorer prognosis with increased EZH2-positive staining and suggests prostate cancer progression and metastasis with increased EZH2 expression (Varambally et al, 2002). In addition to prostate cancer, we have demonstrated that EZH2 protein levels were strongly correlated with breast cancer aggressiveness and promote neoplastic transformation of breast epithelial cells (Kleer et al, 2003). We have also shown that EZH2 promotes anchorageindependent growth and cell invasion and endows primary cells with a proliferative advantage. Additionally, its gene locus is specifically amplified in several primary tumours (Sudo et al, 2005; Bachmann et al, 2006). Importantly, studies have demonstrated the intrinsic enzymatic activity of EZH2 as a histone $\mathrm{H} 3$ lysine 27 methyltransferase, which is critical to its function as a transcriptional repressor and oncogene (Cao and Zhang, 2004). Thus, if a small molecule inhibitor can be identified against EZH2 enzymatic activity or its protein-protein interactions, this may have utility as a viable therapeutic against tumours expressing high levels of EZH2. Based on the early success of HDAC inhibitors, we expect that targeting EZH2 may be a more specific and rational therapy.

Another protein, alpha-methylacyl-CoA racemase (AMACR), is a peroxisomal and mitochondrial enzyme involved in the $\beta$ oxidation of branched fatty acids. It was discovered to have specifically increased expression in prostate cancer epithelia (Rubin et al, 2002). Our studies have also demonstrated that AMACR enzymatic activity is increased in prostate cancer relative to benign epithelia (Figure 1) (Kumar-Sinha et al, 2004). Of particular interest, we have identified a humoral immune response against this enzyme in prostate cancer patient serum (Sreekumar et al, 2004). Furthermore, we have shown that AMACR expression is highest in localised prostate cancer and decreases in metastatic disease, and subsequent reductions in AMACR expression in the former is associated with an increased rate of biochemical
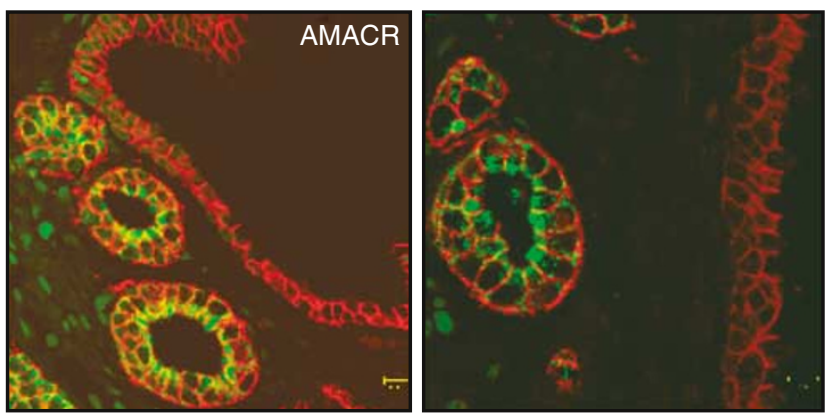

Figure I Prostate cancer tissue staining for AMACR. High levels of the prostate cancer biomarker AMACR (green) stained mainly in clinically localised prostate cancer glands compared with internal adjacent benign glands. A higher magnification image is included on the right, and membrane E-cadherin expression is shown in red. recurrence (Rubin et al, 2005). Although this reduction in hormone-refractory metastasis has been repeatedly observed, little correlation between it and other clinical parameters exists in prostate cancer progression. Additionally, its independence of androgen receptor-mediated signalling has spawned associations between AMACR protein expression loss and tumour dedifferentiation, and the latter's appropriation of pathway control to maintain the formers enzymatic and energy regulation activity. Aside from these observations, a causal link between AMACR protein expression patterns and prostate carcinogenesis is yet to be elucidated.

On the other hand, the centrosome-associated oncogenic kinase Aurora-A, or STK15, belongs to a family of serine/threonine kinases. It has been implicated in playing a crucial role in the control of mitosis. Overexpression of STK15 results in chromosomal aberration, genomic instability and tumorigenesis. This kinase is also known to be amplified in a number of human cancers and tumour cell lines (Jeng et al, 2004). STK15 was also shown to regulate the $\mathrm{p} 53$ pathway by inducing increased degradation of p53, leading to aberrant checkpoint responses and facilitating oncogenic transformation of cells (Katayama et al, 2004). It also plays a key role in G2/M-phase progression. Studies demonstrated that STK15 also provides drug resistance, and inhibiting its expression by RNA interference can result in potent sensitisation to the chemotherapeutic Taxol in human cancer cells (Hata et al, 2005). Our proteomic screen showed a marked upregulation of this kinase in metastatic prostate tumours.

Another protein, MSH2, is encoded for by a gene that is a member of a family of genes long implicated in oncogenesis, and specifically hereditary and sporadic colorectal carcinomas. The gene functions in mismatch recognition during the repair of errors occurring during DNA replication. Identified germ-line missense mutations are known to contribute to the onset of HNPCC, and the gene was shown to be upregulated in primary prostate cancer (Velasco et al, 2002), a result concordant with its protein expression in our screen. The same study also demonstrated that MSH2 expression in prostate carcinoma may be a useful prognostic marker for outcome in men with clinically organconfined prostate carcinoma.

Finally, BM28, whose alias is MCM2, is a well-characterised mini-chromosome maintenance protein, and is known to play an essential role in initiation and regulation of eukaryotic DNA replication. It has been shown to be dysregulated in malignant prostate glands (Meng et al, 2001). The same study also suggested that BM28 expression is an independent predictor of disease-free survival after definitive local therapy, and has potential as a molecular marker for clinical outcome in prostate cancer.

These five either established or putative proteomic alterations in prostate cancer only partially reflect a true diversity of function that reaffirms its molecular heterogeneity. Nonetheless, much remains to be performed both experimentally and bioinformatically for these and many more in pathway enrichment, identifying functional targets, regulatory relationships and protein-protein interaction sub-networks to properly contextualise these entities and understand their contribution to prostate cancer progression.

\section{PROVIDING FUNCTIONAL CONTEXT TO ALTERATIONS}

The molecular profiling of prostate cancer tissue for proteomic alterations has certainly advanced the list of targets potentially mediating the neoplastic phenotype and aggressive subtypes. However, systematic biological contextualisation has lagged their initial discovery. This might be expected as a result of labourintensive experimental characterisation for single proteins, but this also requires mining and computational approaches that can better 
filter these compendiums, annotated and enriched with their corollary data types, much like those developed for the gene expression domain.

An effective repository and analytical platform providing metaanalysis of proteomic alterations in patient material paralleling the successful efforts in cancer microarray analysis would greatly benefit the cancer proteomics community (Rhodes et al, 2004). The task of useful integration and compendium development is complicated by the dimensionality and diversity of proteomic data as previously discussed, which is a more tractable problem in the DNA microarray domain. Despite distinct experimental platforms, DNA microarrays generate uniformly single-dimensional, semiquantitative molecular abundance data. However, proteomic data are decidedly higher dimensional, whose diversity of data type includes quantitative, nominal, categorical, binary and more. Nevertheless, the benefits of such a compendium-based metaanalysis at the protein level, a platform that systematises and integrates diverse protein data from the public domain, are manyfold. They include functional enrichment for biological processes, molecular function, and pathway membership. Another is mapping to existing protein-protein interaction data, and integration for targets of existing therapeutics and small-molecule inhibitor libraries to suggest altered combinatorial therapies at the protein level. Others include integration with antibody repositories, and of course with gene expression data, helping the community better understand the regulatory processes mediating the relative discordance witnessed between the two observed in small scale in previous studies.

We foresee such a platform addressing a variety of current complications in extracting truly causal proteomic alterations from larger, noisy data sets. We briefly identify several problems that would be aided by such an analytical approach. First, and particularly problematic, are those stochastic events that are consequences of the neoplastic phenotype as opposed to determinants of it. Filtering for putative alterations in prostate cancer pathogenesis may begin with altered protein expression, but much like at the transcriptome level, this must be qualified and validated.
A second problem is that of tissue specificity, sample selection and annotation. Certainly one of the primary considerations in this context is how to deal with protein markers having tissue specificity. This trait is indicative of the physiological site of metastatic sample analysed, and is indicative only of the latest stages of secondary-site micro- and macro-metastases and perhaps not causal in progression to metastatic disease. Better bioinformatic tools are required to either properly classify these or subtract them from a causal compendium. Such an analytical platform for comparative analysis across large proteomic data sets culled from a variety of distal sites to the primary site of disease will allow for easier assessment of tissue-specific proteomic noise.

A third important distinction to be made is between those protein markers having utility in a prognostic signature and those with actual biological relevance to the disease state, as these are certainly not mutually inclusive. Correlations between entities, be they genes in a transcriptomic signature, or proteins in proteomic ensemble, may drive the value of a given prognostic signature and a priori outcome determination, but do not equate to fundamental biological interactions, whether regulatory, binding, kinetic or otherwise. These artefacts are often the consequence of a variety of statistical learning implementations that range from 'black box', which masks the structure of the data, to the relatively transparent from which rules can be read with relative ease. However, discriminating between statistical $v s$ biological significance remains a fundamental challenge even with the increasing complexity and sophistication of these analytical approaches.

Many of these complications in generating biological context for proteomic alterations implicated in prostate cancer progression are being addressed through smaller-scale integrative approaches that correlate transcriptomic and proteomic experimental data, mine publicly available data sets, and add dimensionality through the inclusion of molecular interaction data. Integrative biology of this type is paradigmatic of a movement towards a systems biology framework that was previously unavailable to biomedical research (Hood et al, 2004). We foresee that such a compendium for widespread analysis will facilitate these types of analyses on a far larger scale.

\begin{tabular}{l|l|lll|l} 
Sample stratification & Molecular screen & Analytical/computational approach & Outcome
\end{tabular}

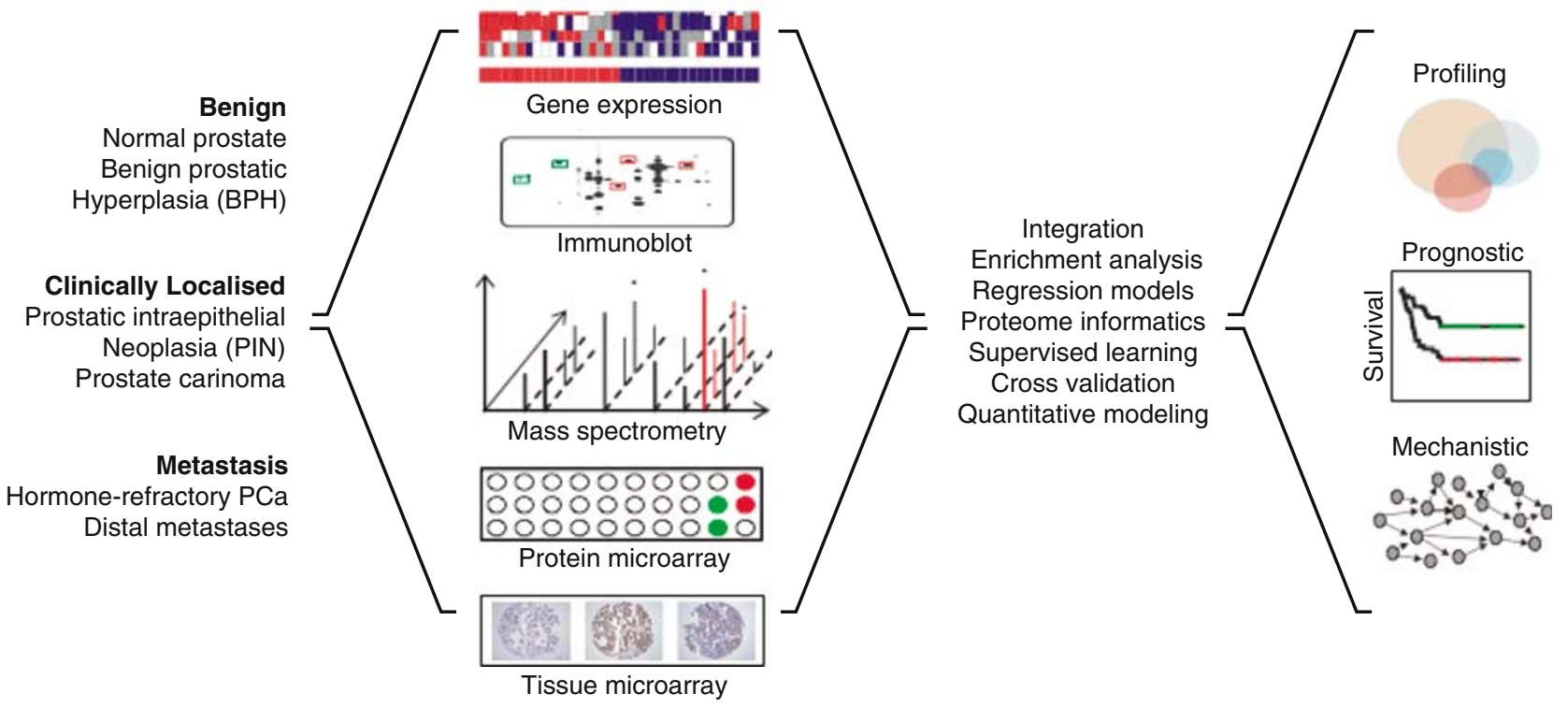

Figure 2 Overview of the conceptual flow in characterising proteomic alterations between prostate cancer stages. Starting from a diverse sample population, a vast array of sample preparation methods and high-throughput technologies can be leveraged to differentially profile tissue stratified by disease progression. A variety of resulting data types, formats, and dimensionality require significant integration and bioinformatic analyses to tease causal entities from the molecular noise and agglomerate the results into one of many desired outcome models motivated by study design. 


\section{CONCLUSION}

As technologies and analytical approaches to prostate cancer profiling mature, so will the compendium of proteomic alterations between stages of disease. However, the contextualisation of these molecular determinants in the biology of cancer progression is fundamental to our understanding of their role in the acquisition of phenotypic hallmarks of individual disease. While complex in implementation and variable in capacity for different sample types, high-throughput technologies being used today are key to this effort. Platforms include mass spectrometry and its corollary sample reduction methods, high-throughput immunoblots, tissue microarrays, and protein microarrays. The latter includes reversephase implementations, antibody arrays, phage display or others in profiling of serum, amplified immune response and others (Figure 2). This broad sampling of platforms is aiding the characterisation of causative alterations at the protein level that are otherwise masked by the molecular noise or lost to physiological variability. However, these are only tools, and their utility must be informed by a comprehensive understanding of the heterogeneous nature of prostate cancer. In larger profiling studies, end-stage metastatic prostate cancer is often aggregated as a single class of disease; however, diverse metastases demonstrate significant heterogeneity, distal organ specificity, and at the molecular level, equate to distinct subtypes of hormonerefractory prostate cancer (Shah et al, 2004). An appreciation for this composition of metastatic disease must filter down to the study of proteomic alterations, not simply stratified by progression through discrete disease stages, but also within-stage subtypes. This approach promises an alternative to end-point analysis, rather a dynamic proteomic model of progression to metastatic prostate cancer, a combinatorial ensemble suitable for efforts ranging from quantitative modelling to therapeutic perturbation.

\section{ACKNOWLEDGEMENTS}

This research was supported in part by the Department of Defense (PC051081 to AMC and SV), the American Cancer Society (RSG02-179-MGO to AMC), the National Institutes of Health (RO1 CA CA97063, Prostate SPORE P50CA69568 to AMC and SV; and Early Detection Research Network UO1 CA111272-01 to AMC) and the UM Cancer Center Support Grant (5P30 CA46592) funding to the UMCCC Bioinformatics Core.

\section{REFERENCES}

Bachmann IM, Halvorsen OJ, Collett K, Stefansson IM, Straume O, Haukaas SA, Salvesen HB, Otte AP, Akslen LA (2006) EZH2 expression is associated with high proliferation rate and aggressive tumor subgroups in cutaneous melanoma and cancers of the endometrium, prostate, and breast. J Clin Oncol 24: 268-273

Cao R, Zhang Y (2004) The functions of E(Z)/EZH2-mediated methylation of lysine 27 in histone H3. Curr Opin Genet Dev 14: 155-164

Caprioli RM (2005) Deciphering protein molecular signatures in cancer tissues to aid in diagnosis, prognosis, and therapy. Cancer Res 65: $10642-10645$

Dan HC, Sun M, Kaneko S, Feldman RI, Nicosia SV, Wang HG, Tsang BK, Cheng JQ (2004) Akt phosphorylation and stabilization of X-linked inhibitor of apoptosis protein (XIAP). J Biol Chem 279: $5405-5412$

Dhanasekaran SM, Barrette TR, Ghosh D, Shah R, Varambally S, Kurachi K, Pienta KJ, Rubin MA, Chinnaiyan AM (2001) Delineation of prognostic biomarkers in prostate cancer. Nature 412: $822-826$

Hata T, Furukawa T, Sunamura M, Egawa S, Motoi F, Ohmura N, Marumoto T, Saya H, Horii A (2005) RNA interference targeting aurora kinase a suppresses tumor growth and enhances the taxane chemosensitivity in human pancreatic cancer cells. Cancer Res 65: 2899-2905

Hood L, Heath JR, Phelps ME, Lin B (2004) Systems biology and new technologies enable predictive and preventative medicine. Science 306: $640-643$

Hopfner R, Mousli M, Jeltsch JM, Voulgaris A, Lutz Y, Marin C, Bellocq JP, Oudet P, Bronner C (2000) ICBP90, a novel human CCAAT binding protein, involved in the regulation of topoisomerase IIalpha expression. Cancer Res 60: $121-128$

Jammi NV, Beal PA (2001) Phosphorylation of the RNA-dependent protein kinase regulates its RNA-binding activity. Nucleic Acids Res 29: 3020 3029

Jeng YM, Peng SY, Lin CY, Hsu HC (2004) Overexpression and amplification of Aurora-A in hepatocellular carcinoma. Clin Cancer Res 10: 2065-2071

Katayama H, Sasai K, Kawai H, Yuan ZM, Bondaruk J, Suzuki F, Fujii S, Arlinghaus RB, Czerniak BA, Sen S (2004) Phosphorylation by aurora kinase A induces Mdm2-mediated destabilization and inhibition of p53. Nat Genet 36: 55-62

Kleer CG, Cao Q, Varambally S, Shen R, Ota I, Tomlins SA, Ghosh D, Sewalt RG, Otte AP, Hayes DF, Sabel MS, Livant D, Weiss SJ, Rubin MA, Chinnaiyan AM (2003) EZH2 is a marker of aggressive breast cancer and promotes neoplastic transformation of breast epithelial cells. Proc Natl Acad Sci USA 100: 11606-11611

Kumar-Sinha C, Chinnaiyan AM (2003) Molecular markers to identify patients at risk for recurrence after primary treatment for prostate cancer. Urology 62(Suppl 1): 19-35

Kumar-Sinha C, Shah RB, Laxman B, Tomlins SA, Harwood J, Schmitz W, Conzelmann E, Sanda MG, Wei JT, Rubin MA, Chinnaiyan AM (2004) Elevated alpha-methylacyl-CoA racemase enzymatic activity in prostate cancer. Am J Pathol 164: 787-793

Ludwig JA, Weinstein JN (2005) Biomarkers in cancer staging, prognosis and treatment selection. Nat Rev Cancer 5: 845-856

Meng MV, Grossfeld GD, Williams GH, Dilworth S, Stoeber K, Mulley TW, Weinberg V, Carroll PR, Tlsty TD (2001) Minichromosome maintenance protein 2 expression in prostate: characterization and association with outcome after therapy for cancer. Clin Cancer Res 7: $2712-2718$

Poukka H, Aarnisalo P, Karvonen U, Palvimo JJ, Janne OA (1999) Ubc9 interacts with the androgen receptor and activates receptor-dependent transcription. J Biol Chem 274: $19441-19446$

Rhodes DR, Yu J, Shanker K, Deshpande N, Varambally R, Ghosh D, Barrette T, Pandey A, Chinnaiyan AM (2004) ONCOMINE: a cancer microarray database and integrated data-mining platform. Neoplasia 6: $1-6$

Rubin MA, Bismar TA, Andren O, Mucci L, Kim R, Shen R, Ghosh D, Wei JT, Chinnaiyan AM, Adami HO, Kantoff PW, Johansson JE (2005) Decreased alpha-methylacyl CoA racemase expression in localized prostate cancer is associated with an increased rate of biochemical recurrence and cancer-specific death. Cancer Epidemiol Biomarkers Prev 14: $1424-1432$

Rubin MA, Zhou M, Dhanasekaran SM, Varambally S, Barrette TR, Sanda MG, Pienta KJ, Ghosh D, Chinnaiyan AM (2002) alpha-Methylacyl coenzyme A racemase as a tissue biomarker for prostate cancer. JAMA 287: $1662-1670$

Santagata S, Demichelis F, Riva A, Varambally S, Hofer MD, Kutok JL, Kim R, Tang J, Montie JE, Chinnaiyan AM, Rubin MA, Aster JC (2004) JAGGED1 expression is associated with prostate cancer metastasis and recurrence. Cancer Res 64: 6854-6857

Shah RB, Mehra R, Chinnaiyan AM, Shen R, Ghosh D, Zhou M, Macvicar GR, Varambally S, Harwood J, Bismar TA, Kim R, Rubin MA, Pienta KJ (2004) Androgen-independent prostate cancer is a heterogeneous group of diseases: lessons from a rapid autopsy program. Cancer Res 64: $9209-9216$

Sreekumar A, Laxman B, Rhodes DR, Bhagavathula S, Harwood J, Giacherio D, Ghosh D, Sanda MG, Rubin MA, Chinnaiyan AM (2004) Humoral immune response to alpha-methylacyl-CoA racemase and prostate cancer. J Natl Cancer Inst 96: 834-843 
Stanbrough M, Bubley GJ, Ross K, Golub TR, Rubin MA, Penning TM, Febbo PG, Balk SP (2006) Increased expression of genes converting adrenal androgens to testosterone in androgen-independent prostate cancer. Cancer Res 66: 2815-2825

Sudo T, Utsunomiya $\mathrm{T}$, Mimori $\mathrm{K}$, Nagahara $\mathrm{H}$, Ogawa $\mathrm{K}$, Inoue $\mathrm{H}$, Wakiyama S, Fujita H, Shirouzu K, Mori M (2005) Clinicopathological significance of EZH2 mRNA expression in patients with hepatocellular carcinoma. Br J Cancer 92: 1754-1758

Varambally S, Dhanasekaran SM, Zhou M, Barrette TR, Kumar-Sinha C, Sanda MG, Ghosh D, Pienta KJ, Sewalt RG, Otte AP, Rubin MA, Chinnaiyan AM (2002) The polycomb group protein EZH2 is involved in progression of prostate cancer. Nature 419: 624-629

Varambally S, Yu J, Laxman B, Rhodes DR, Mehra R, Tomlins SA, Shah RB, Chandran U, Monzon FA, Becich MJ, Wei JT, Pienta KJ, Ghosh D, Rubin MA, Chinnaiyan AM (2005) Integrative genomic and proteomic analysis of prostate cancer reveals signatures of metastatic progression. Cancer Cell 8: $393-406$
Velasco A, Hewitt SM, Albert PS, Hossein M, Rosenberg H, Martinez C, Sagalowsky AI, McConnell JD, Marston W, Leach FS (2002) Differential expression of the mismatch repair gene hMSH2 in malignant prostate tissue is associated with cancer recurrence. Cancer 94: $690-699$

Vire E, Brenner C, Deplus R, Blanchon L, Fraga M, Didelot C, Morey L, Van Eynde A, Bernard D, Vanderwinden JM, Bollen M, Esteller M, Di Croce L, de Launoit Y, Fuks F (2006) The Polycomb group protein EZH2 directly controls DNA methylation. Nature 439: $871-874$

Watanabe M, Fukutome K, Kato H, Murata M, Kawamura J, Shiraishi T, Yatani R (1999) Progression-linked overexpression of c-Met in prostatic intraepithelial neoplasia and latent as well as clinical prostate cancers. Cancer Lett 141: $173-178$

Wu CC, Yates III JR (2003) The application of mass spectrometry to membrane proteomics. Nat Biotechnol 21: 262-267

Wulfkuhle JD, Liotta LA, Petricoin EF (2003) Proteomic applications for the early detection of cancer. Nat Rev Cancer 3: 267-275 